\title{
Dissecting the cryoprotection mechanisms for dehydrins
}

\section{Cesar L. Cuevas-Velazquez, David F. Rendón-Luna and Alejandra A. Covarrubias*}

Departamento de Biología Molecular de Plantas, Instituto de Biotecnología, Universidad Nacional Autónoma de México, Cuernavaca, México

\section{Edited by:}

Steffen P. Graether, University of

Guelph, Canada

\section{Reviewed by:}

Vasileios Fotopoulos, Cyprus

University of Technology, Cyprus

Saman Seneweera, The University of

Melbourne, Australia

Steffen P. Graether, University of

Guelph, Canada

*Correspondence:

Alejandra A. Covarrubias,

Departamento de Biología Molecular

de Plantas, Instituto de Biotecnología, Universidad Nacional Autónoma de

México, Avenida Universidad 2001,

Colonia Chamilpa, 62210 Cuernavaca,

Morelos, México

e-mail:crobles@ibt.unam.mx
One of the common responses of plants to water deficit is the accumulation of the socalled late embryogenesis abundant (LEA) proteins. In vitro studies suggest that these proteins can protect other macromolecules and cellular structural components from the impairments caused by water limitation. Their binding to phospholipids, nucleic acids and/or to divalent cations has suggested multi-functionality. Genetic analyses indicate that these proteins are required for an optimal adjustment of plants to this insult. This diverse information has conducted to propose different models for LEA proteins action mechanisms. Many of these properties are shared by group 2 LEA proteins or dehydrins (DHNs), one of the LEA protein families for which large amount of data is available. This manuscript focuses on the different mechanisms proposed for this LEA protein group by analyzing published data derived from in vitro cryoprotection assays. We compared the molar ratio of protectant:enzyme needed to preserve $50 \%$ of the initial activity per enzyme monomer to assess different mechanisms of action. Our results add evidence for protein-protein interaction as a protection mechanism but also indicate that some DHNs might protect by different means. The strength and weakness of the proposed protection mechanisms are discussed.

Keywords: dehydrins, late embryogenesis abundant proteins, cryoprotection, water deficit, abiotic stress, intrinsically disordered proteins

\section{DEHYDRINS, A PLANT SPECIFIC GROUP OF LEA PROTEINS}

Late embryogenesis abundant (LEA) proteins are a group of enigmatic proteins that have been strongly associated with plant responses to water deficit (Battaglia et al., 2008; Hincha and Thalhammer, 2012). They accumulate mainly in dry seeds but also in vegetative tissues when plants experience water deficit such as drought, freezing and high salinity. Among the different LEA proteins, group 2 LEA proteins (D11) have been the most studied (Rorat, 2006; Hara, 2010; Hanin et al., 2011). Proteins in this group are also known as dehydrins (DHNs), and to date they have been found only in plants (Battaglia et al., 2008; Hara, 2010). Due to the vast majority of DHNs reports over other LEA proteins, people not familiar with these proteins assume that all LEA proteins are DHNs; however, this is not the case, LEA proteins represent a rather large group of diverse proteins. Depending on sequence similarity and the presence of particular motifs, LEA proteins have been classified in at least seven groups or families (Battaglia et al., 2008). Although there is sequence similarity within proteins in each group, different LEA protein groups exhibit virtually no sequence conservation with each other. Nevertheless, typical LEA proteins share distinctive physicochemical characteristics such as high hydrophilicity, high content of Gly, Ala and Ser, and lack or underrepresentation of Cys, Trp, and other hydrophobic amino acids (Dure, 1993; Garay-Arroyo et al., 2000; Hundertmark and Hincha, 2008). These characteristics suggest that LEA proteins from different groups might have similar functional properties.

Computational analyses have indicated that, as other LEA proteins, DHNs lack stable tridimensional structures, leading to be considered as intrinsically disordered proteins (IDPs; Close, 1996;
Battaglia et al., 2008). Some of them have been experimentally characterized as IDPs in solution (Ismail et al., 1999; Soulages et al., 2003; Bokor et al., 2005). In spite of this structural flexibility, it has been shown that some DHNs gain ordered structure in the presence and binding to other molecules such as lipids, nucleic acids, or metal ions (Koag et al., 2003; Hara et al., 2009; Eriksson et al., 2011; Rahman et al., 2011).

Dehydrins have been defined by the presence of a Lys-rich segment, or K-segment, which may be repeated several times. Moreover, they are further classified in sub-classes depending on the representation of some of their distinctive conserved motifs, such as the Y-segment (Tyr-rich), or the S-segment (Ser-track). Accordingly, five sub-classes can be distinguished: $\mathrm{K}_{(\mathrm{n})}, \mathrm{SK}_{(\mathrm{n})}$ $\mathrm{K}_{(\mathrm{n})} \mathrm{S}, \mathrm{Y}_{(\mathrm{n})} \mathrm{K}_{(\mathrm{n})}$, and $\mathrm{Y}_{(\mathrm{n})} \mathrm{SK}_{(\mathrm{n})}$ ( $\mathrm{n}=$ number of repeats), for each of which different functions have been proposed (Hara, 2010; Hanin et al., 2011).

Several approaches have been followed to determine the function of these proteins, using in vivo and in vitro experimental systems. Even though for some LEA protein groups it has been shown their participation in the response of vascular plants to water limiting environments by reverse genetics (Manfre et al., 2006; Kim and Nam, 2010; Olvera-Carrillo et al., 2010), this has not been the case for DHNs, mostly due to the large number of members found within this family (Arabidopsis group 2 LEA proteins presents 10 members; Battaglia et al., 2008; Hundertmark and Hincha, 2008). However, a contribution to salt and osmotic stress tolerance was reported for the two DHN genes (PpDHNA and $P p D H N B)$ of the moss Physcomitrella patens, for which targeted knockout mutants were characterized (Saavedra et al., 2006; Ruibal et al., 2012). Binding to other macromolecules such as 
negatively charged lipids and DNA (Koag et al., 2003; Hara et al., 2009) have led to propose that DHNs can protect the integrity of biological membranes and nucleic acids from the effects caused by low water availability. Also, the binding to divalent cations such as $\mathrm{Ca}^{2+}, \mathrm{Zn}^{2+}, \mathrm{Fe}^{3+}, \mathrm{Co}^{2+}, \mathrm{Ni}^{2+}$, and $\mathrm{Cu}^{2+}$ has suggested that DHNs might act as buffer for these metals under water deficit (Kruger et al., 2002; Alsheikh et al., 2005; Hara et al., 2005). The ability of DHNs to bind to such diverse set of ligands could be due to their structural flexibility.

An extensively explored possibility for DHNs function has been their competence to protect other proteins from the effects resulting from water scarcity in the cellular environment. This hypothesis has been addressed by in vitro assays, where water limitation is imposed by partial water dehydration or by freeze/thaw cycles. The results from these experiments have demonstrated that different DHNs can prevent the inactivation of reporter enzymes [lactate dehydrogenase (LDH); alcohol dehydrogenase $(\mathrm{ADH})$; firefly luciferase; citrate synthase $(\mathrm{CS}) ; \beta$-glucosidase $\mathrm{G}$, $(\beta g l G)$; and glucose oxidase (GOD/POD)] under these different water deficit conditions indicating that some of them are cryoand/or dehydro-protectors (Sanchez-Ballesta et al., 2004; Tantos et al., 2009; Brini et al., 2010; Drira et al., 2013).

\section{DISSECTING THE MOLECULAR MECHANISM OF DHNS PROTEIN PROTECTION}

Several years ago, it was reported that addition of some LEA proteins, including DHNs, prevented the inactivation of reporter enzymes upon various freezing and thawing cycles (Lin and Thomashow, 1992; Kazuoka and Oeda, 1994). This in vitro assay is now a common approach to evaluate the protective activity of LEA proteins under these stress condition (Wisniewski et al., 1999; Hara etal., 2001; Bravo et al., 2003; Momma et al., 2003; Sanchez-Ballesta etal., 2004; Reyes etal., 2008; Hughes and Graether, 2011; Hughes etal., 2013). Kazuoka and Oeda (1994) reported that SoCOR85, an 85 KDa DHN from spinach, was able to keep LDH activity after freezing the sample for $24 \mathrm{~h}$ at $-20^{\circ} \mathrm{C}$ and subsequent thawing at $4^{\circ} \mathrm{C}$ for $2 \mathrm{~h}$. In that work, SoCOR85 showed a $\mathrm{PD}_{50}$ ("Protectant protein" concentration needed to preserve $50 \%$ of the reporter enzyme activity) smaller than that for a variety of proteins with no relation to DHNs, including BSA, a known cryoprotectant. These data indicated that SoCOR85 was an effective cryoprotectant, and suggested that this cryoprotective activity was specific (Kazuoka and Oeda, 1994). Subsequently, a similar activity was found for DHNs from different plant species such as TaWCS120 from wheat (Houde et al., 1995), PpPCA60 from peach (Wisniewski et al., 1999), CuCOR19 from Citrus unshiu (Hara etal., 2001), HvP-80/Dhn5 from barley (Bravo et al., 2003), GmDHN26 and GmDHN27 from soybean (Momma et al., 2003), CrCOR15 from fortune mandarin fruit (Sanchez-Ballesta et al., 2004), ERD10 from Arabidopsis (Reyes et al., 2008), RcDhn5 from Rhododendron catawbiense Michaux (Reyes et al., 2008), ERD14 from Arabidopsis (Tantos et al., 2009), TaDHN-5 from wheat (Drira et al., 2013), VrYSK $_{2}$ from Vitis riparia (Hughes and Graether, 2011; Hughes et al., 2013), TsDHN-2 from Thellungiella salsuginea (Hughes et al., 2013), and OpsDHN-1 from Opuntia streptacantha (Hughes et al., 2013).
Although there are some differences in the in vitro assays cited above (Supplemental Table 1), the data from this experimental system seem to be a good platform to compare the effectiveness of this activity among the different DHNs tested, and to address questions regarding the mechanism of action involved in such protecting effect. In order to be able to compare the data from these different reports, we have unified this information by considering not only the amount but also the molecular mass of the proteins assayed; hence, instead of comparing $\mathrm{PD}_{50}$ we compared the molar ratio of protectant:enzyme, which ponders the amount of molecules needed to preserve $50 \%$ of the initial activity per monomer of the reporter enzyme (Molar Ratio $50, \mathrm{MR}_{50}$ ). $\mathrm{LDH}$ was the common reporter enzyme in all cases analyzed here. The original and standardized data are shown in Supplemental Table 1. The comparison between the $\mathrm{MR}_{50}$ of the different DHNs showed a broad range of cryoprotection levels, from 0.05 to 66.5. Hv80/Dhn5 showed to be the most effective cryoprotectant with a $\mathrm{MR}_{50}$ of 0.07 indicating that only one molecule (or less) of DHN is required to protect one molecule of $\mathrm{LDH}$ monomer during freeze/thaw treatments (Figure 1A). Even considering TsDHN2, the less effective protectant with a $\mathrm{MR}_{50}$ of 66.5 , DHNs cryoprotection effectiveness seems remarkable when it is compared with that attained by compatible osmolytes such as sucrose, for which $10^{6}$ molecules are needed to protect one monomer of $\mathrm{LDH}$ (Houde et al., 1995).

This analysis allows envisaging at least two different molecular mechanisms of action to explain DHNs protective activity, both already proposed in different reports (Reyes et al., 2005; Tunnacliffe and Wise, 2007; Tompa and Kovacs, 2010; Olvera-Carrillo et al., 2011; Chakrabortee et al., 2012; Hughes et al., 2013). One, supported by the low DHN:enzyme molar ratios needed to get protection, in which protein-protein interaction is strongly suggested, and another where a higher amount of DHN molecules seem to be necessary to exert this protective effect (Figures 1A and 2). The first hypothesis is also supported by additional published data that was not considered in this study because the available data did not allow estimating $\mathrm{MR}_{50}$ values. This is the case for ERD10, an Arabidopsis DHN, which was able to keep $75 \%$ of LDH activity after five freeze/thaw cycles in a 1:1 (ERD10:LDH) molar ratio, suggesting a $\mathrm{MR}_{50}$ lower than 1 (Reyes et al., 2008). Similarly, ERD14 from Arabidopsis preserved $100 \%$ of the ADH activity when subjected to five freeze/thaw cycles in a 0.5:1 (ERD14:LDH) molar ratio (Tantos et al., 2009).

Those cases with a high $\mathrm{MR}_{50}$, where many DHN molecules are required for cryoprotection, suggest a mechanism where DHN molecules would not necessarily need to contact the cryosusceptible target protein but rather stay localized near the enzyme and, because their hydrophilic and highly disordered characteristics, they may provide an appropriate environment to stabilize a native and functional structure, a mechanism that has been referred as molecular shield (Tunnacliffe and Wise, 2007; Chakrabortee et al., 2012; Hughes et al., 2013). For this last mechanism, it has been proposed that the extended random coil structure reported for some of the characterized DHNs would result in proteins with a large hydrodynamic radius, which predicts that DHNs with this conformation would be able to align their hydrophilic amino acids around the surface of a target protein to circumvent 


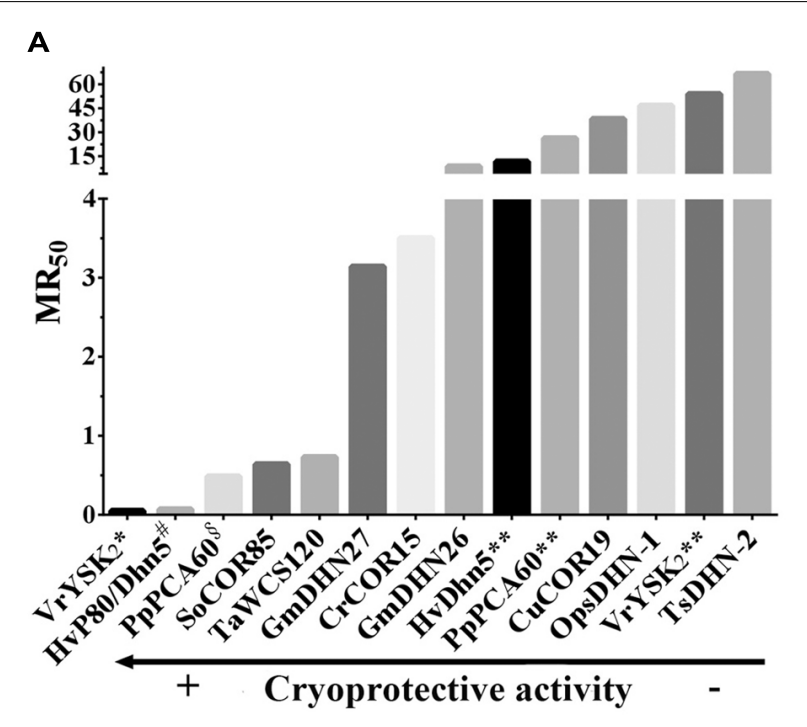

B

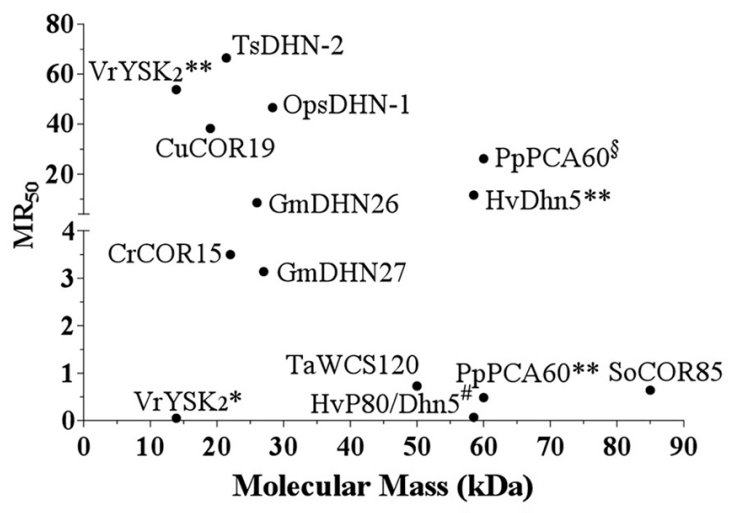

FIGURE 1 | (A) Cryoprotective efficiency of DHNs. The DHN considered for this analysis were those for which the available data allowed to calculate the amount of molecules needed to preserve $50 \%$ of the initial activity per molecule of the reporter enzyme or molecular ratio $50\left(M_{50}\right)$. The data used for these calculations and the resulting $\mathrm{MR}_{50}$ values are shown in Supplementary Table 1. (B) $M_{50}$ values for $\mathrm{DHNs}$ with different molecular mass. A conflict in the reported data leading to contrasting cryoprotective efficiency was found in the following cases: VrYSK2* (Hughes and Graether, 2011), VrYSK2** (Hughes etal., 2013), PpPCA60§ Misniewski et al., 1999), PpPCA60** (Hughes etal., 2013), HvDhn5** (Hughes et al., 2013), and HvP80/Dhn5\# (Bravo etal., 2003). MR ${ }_{50}$ values were calculated using the following equation: $\mathrm{MR}_{50}=\left\{(36.4) * \mathrm{PD}_{50}\right\} /\left\{[\mathrm{LDH}]^{*} \mathrm{MM}\right\}$, where 36.4 is the molecular mass of $\mathrm{LDH}$ monomer in $\mathrm{kDa}$; $\mathrm{PD}_{50}$ in $\mu \mathrm{g} / \mathrm{mL}$; [LDH]: LDH concentration in $\mu \mathrm{g} / \mathrm{mL}$; MM: Dehydrin's molecular mass.

the loss of its bulk water and consequent changes in its native structure (Tunnacliffe and Wise, 2007; Hughes et al., 2013). This hypothesis is supported by a recent work, where different concatemers of the $\mathrm{K}_{2}$ hypothetical protein from $V$. riparia were used (Hughes et al., 2013). In this report it was found that the level of protection conferred by these K-segment concatemers was directly proportional to their hydrodynamic radius. A similar correlation was observed by comparing the protection level of polyethylene glycol (PEG) polymers with different hydrodynamic radius. By contrast, no correlation was detected when globular proteins were
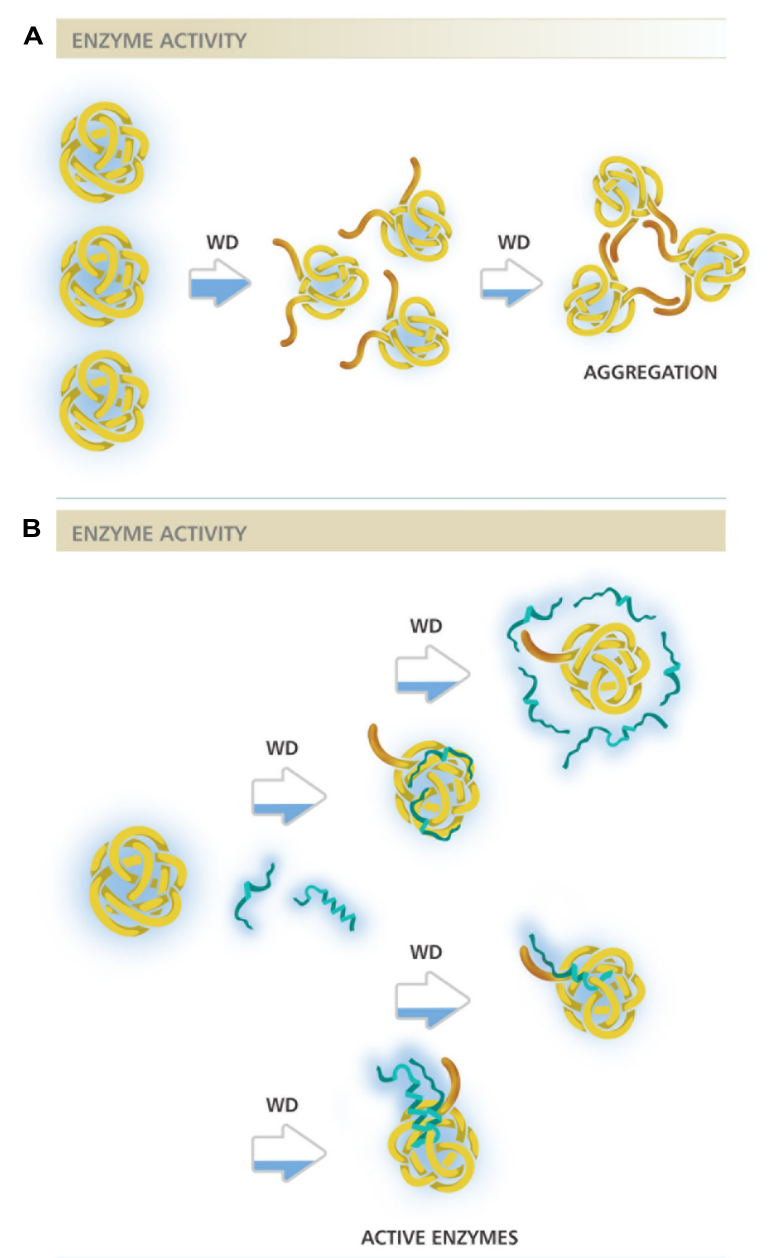

FIGURE 2 | Different molecular mechanisms proposed for DHNs protective activity. (A) Representation of an enzyme under water deficit in the absence of DHNs. Brown extensions represent enzyme hydrophobic regions starting to be exposed as a consequence of low water availability, indicated by the partially blue filled arrows. A severe dehydration may lead to a higher exposure of hydrophobic regions resulting in enzyme aggregation. (B) Representation of an enzyme under water deficit in the presence of DHNs. Blue strands represent DHN molecules in two possible structural conformations, with high structural disorder and with some helicity possibly gained under water limitation. The different mechanisms proposed are represented after each partially blue filled arrow. From top to bottom: the first two figures illustrate protection by molecular shield, where DHN molecules not necessarily interact with their targets but rather their proximity maintain the amount and organization of water molecules needed to keep enzyme integrity. A variant of this mechanism considers their hydrodynamic radius, a highly disordered DHN molecule could present a large hydrodynamic radius able to cover a larger surface of its target; the two bottom figures represent two variants of the mechanism where protein-protein interaction is required to select and protect their targets, in one case DHN can bind as monomers whereas in other as dimer or any other oligomeric form. The filling in the horizontal bars above schemes represents enzyme activity level.

tested (Hughes et al., 2013). However, if we consider the $\mathrm{MR}_{50}$ for these proteins (Supplemental Table 1), they showed values between 39.3 and 668 (up to 668 molecules of DHN are needed to protect one molecule of LDH). From these data, it is evident that 
the cryoprotective efficiency of these concatemers is far low from that showed by other DHNs, where protection was detected even with one protein molecule (Figure 1A; Supplemental Table 1), suggesting that cryoprotection through molecular shield is less efficient than that obtained by protein-protein interaction. The need of a larger number of molecules that could be protecting through a molecular shield mechanism could also be interpreted as an unspecific effect produced just by the presence of many molecules close to a sensitive target, which also could occur by some other proteins apparently not devoted to such specific function. If only the hydrodynamic radius and the hydrophilicity of a protecting protein are relevant for this effect, then any polypeptide with these properties despite their amino acid sequence would be a cryoprotectant, which raises a question remaining to be addressed. On this regard, it has been reported that a large and highly hydrophilic amino acid-based polymer, poly L-Lysine was unable to protect LDH activity from freeze/thaw treatments (Reyes et al., 2008), implying the contribution of additional characteristics in a protein to be a good protectant. The relevance of the protein sequence also has to be considered for the data obtained using the $\mathrm{K}_{2}$ concatemers because the proteins obtained not only are longer with larger hydrodynamic radius but they also contain an increasing number of the conserved K-segments, whose specific sequence could influence on this effect. The participation of K-segments in DHNs cryoprotection was suggested because their progressive deletion from Arabidopsis thaliana and Rhododendron catawbiense DHNs (ERD10 and RcDhn5, respectively) impaired their ability to protect LDH activity (Reyes et al., 2008). Similar findings were obtained for wheat DHN-5 (Drira et al., 2013).

The $\mathrm{MR}_{50}$ from many of the DHN cryoprotection experiments reported also allowed us to look for a relation between the size of natural DHNs and their molecular protection effectiveness; however, no correlation was found between these two parameters (Figure 1B). There are low molecular mass DHNs with an $\mathrm{MR}_{50}$ close to 70 and others whose $\mathrm{MR}_{50}$ was lower than 10. Although some correlation could be detected for the K-segments analyzed by Hughes et al. (2013; see Supplementary Table 1), the data for any set of the natural DHNs, with low or high molecular mass (Figure 1B), are not consistent with a general mechanism in which the length of DHNs plays an important role in cryoprotection. Furthermore, we neither found any correlation by considering the levels of structural disorder determined using PONDR tools (Romero et al., 1997). HvP80/Dhn5 with the highest cryoprotection efficiency shows the same level of disorder (52.7\%) than that obtained for TsDHN2 (52.43\%) with the lowest cryoprotection efficiency, indicating that the protective effect is rather related to specific properties in each of the DHNs tested.

Results where direct binding between a reporter enzyme and a DHN was not detected favored the idea that the physicochemical properties of LEA proteins (including DHNs), such as the abundance of charged amino acid residues, promote electrostatic interactions to keep the two proteins closely enough to provide protection without binding (Hughes and Graether, 2011). However, data indicating that one or few LEA protein molecules are enough to protect reporter enzymes from the effects of water scarcity (Kazuoka and Oeda, 1994; Houde et al., 1995; Bravo et al., 2003; Reyes et al., 2005; Nakayama etal., 2007) sustains the possibility of direct protein interaction, which is further supported by the analysis made in the present work. Moreover, evidence for a physical interaction between LEA proteins and target proteins has been obtained for some hydrophilins and LEA proteins (Alsheikh et al., 2005; Reyes et al., 2005; Nakayama et al., 2007; Kushwaha et al., 2012, 2013; Xie etal., 2012). This has also been the case for $\mathrm{Y}_{2} \mathrm{~K}_{4}$-type $\mathrm{DHN}$ from Medicago truncatula, for which it was found in vitro and in vivo interaction with an ICE-type (inducer of CBF expression 1) transcription factor (Xie etal., 2012). The low occurrence of hydrophobic patches in the many DHNs and other LEA proteins predicts low affinity association with other proteins; however, attention should be given to their amino acid sequence as well as to possible structural modifications that could be promoted by changes in their environment (Mouillon et al., 2008; Olvera-Carrillo et al., 2011) and/or by their interaction with their clients, as it has been proposed for various IDPs (Dyson and Wright, 2005; Tompa and Fuxreiter, 2008; Pancsa and Tompa, 2012). Based on the physicochemical properties of IDPs and some experimental evidence (Pufall et al., 2005; Galea etal., 2008; Wang et al., 2011), it has also been hypothesized that the existence of a variety of structural states for a particular IDP or intrinsically disordered regions (IDRs) could lead to the formation of dynamic protein complexes, where this macromolecular ensembles may fluctuate between diverse structural organizations (Tompa and Fuxreiter, 2008). Hence, protein-protein interactions between LEA protein and their clients may be transient, making more challenging the finding of experimental conditions where these interactions could be stabilized.

\section{FUTURE DIRECTIONS}

Overall, considering the differences in the reported evidence and the analysis in this study, it cannot be discarded that different DHNs perform their protective function by different mechanisms or a combination of them (see Figure 2) depending on their particular sequences or even on the severity of the stress and/or cell type where they carry out their function. The possibility that DHNs perform multiple functions, a feature that seems to be common for IDPs (Jeffery, 2003; Sun et al., 2013), is something to be considered for their molecular mechanisms of action. Evidence for multi-functionality has been obtained in vitro for some DHNs, showing their ability to bind divalent cations, nucleic acids or some phospholipids, in addition to their cryoprotective effect (Koag et al., 2003, 2009; Hanin et al., 2011). It should be kept in mind the possible role of post-translational modifications in the modulation of different functions and interactions, considering a further structural and functional characterization of DHNs obtained from plant tissues. Their potential role in plant cells as protectant proteins or chaperones during water deficit needs additional evidence, identifying their natural targets or clients as well as regions or sequences in their structure relevant for their function.

The structural disordered nature of DHNs, their distinctive properties and sequences impose ad hoc experimental designs, some of them challenging but needed to get closer to the understanding of their function in the plant responses to abiotic and 
biotic stress. It is imperative the analysis of a larger number of DHNs as well as the standardization of in vitro protection assays to be able to address various aspects of the proposed mechanisms. The emergence of new and high-resolution technologies represents a good opportunity to validate the proposed molecular mechanisms and to address their relevance in the plant cell.

\section{ACKNOWLEDGMENTS}

We are grateful to María Teresa Covarrubias for artwork design. This work was partially supported by Consejo Nacional de Ciencia y Tecnología-Mexico (CONACyT-132258) and Programa de Apoyo a Proyectos de Investigación e Innovación Tecnológica (PAPIIT-DGAPA-UNAM; IN-208212). CLC-V and DFR-L were supported by CONACyT with a Ph.D. and MSci fellowships, respectively.

\section{SUPPLEMENTARY MATERIAL}

The Supplementary Material for this article can be found online at: http://www.frontiersin.org/journal/10.3389/fpls.2014.00583/ abstract

\section{REFERENCES}

Alsheikh, M. K., Svensson, J. T., and Randall, S. K. (2005). Phosphorylation regulated ion-binding is a property shared by the acidic subclass dehydrins. Plant Cell Environ. 28, 1114-1122. doi: 10.1111/J.1365-3040.2005.01348.X

Battaglia, M., Olvera-Carrillo, Y., Garciarrubio, A., Campos, F., and Covarrubias, A. A. (2008). The enigmatic LEA proteins and other hydrophilins. Plant Physiol. 148, 6-24. doi: 10.1104/Pp.108.120725

Bokor, M., Csizmok, V., Kovacs, D., Banki, P., Friedrich, P., Tompa, P., et al. (2005). NMR relaxation studies on the hydrate layer of intrinsically unstructured proteins. Biophys. J. 88, 2030-2037. doi: 10.1529/Biophysj.104.051912

Bravo, L. A., Gallardo, J., Navarrete, A., Olave, N., Martinez, J., Alberdi, M., et al. (2003). Cryoprotective activity of a cold-induced dehydrin purified from barley. Physiol. Plant. 118, 262-269. doi: 10.1034/J.1399-3054.2003.00060.X

Brini, F., Saibi, W., Amara, I., Gargouri, A., Masmoudi, K., and Hanin, M. (2010). Wheat dehydrin DHN-5 exerts a heat-protective effect on beta-glucosidase and glucose oxidase activities. Biosci. Biotechnol. Biochem. 74, 1050-1054. doi: 10.1271/Bbb. 90949

Chakrabortee, S., Tripathi, R., Watson, M., Schierle, G. S. K., Kurniawan, D. P., Kaminski, C. F., et al. (2012). Intrinsically disordered proteins as molecular shields. Mol. Biosyst. 8, 210-219. doi: 10.1039/C1mb05263b

Close, T. J. (1996). Dehydrins: emergence of a biochemical role of a family of plant dehydration proteins. Physiol. Plant. 97, 795-803. doi: 10.1111/J.13993054.1996.Tb00546.X

Drira, M., Saibi, W., Brini, F., Gargouri, A., Masmoudi, K., and Hanin, M. (2013). The K-segments of the wheat dehydrin DHN-5 are essential for the protection of lactate dehydrogenase and $\beta$-glucosidase activities in vitro. Mol. Biotechnol. 54, 643-650. doi: 10.1007/S12033-012-9606-8

Dure, L. (1993). "Structural motifs in lea proteins," in Plant Responses to Cellular Dehydration during Environmental Stress, Vol. 10, eds T. J. Close and E. A. Bray (Rockville, MD: American Society of Plant Physiologists), 91-103.

Dyson, H. J., and Wright, P. E. (2005). Intrinsically unstructured proteins and their functions. Nat. Rev. Mol. Cell Biol. 6, 197-208. doi: 10.1038/Nrm1589

Eriksson, S. K., Kutzer, M., Procek, J., Grobner, G., and Harryson, P. (2011). Tunable membrane binding of the intrinsically disordered dehydrin Lti30, a cold-induced plant stress protein. Plant Cell 23, 2391-2404. doi: 10.1105/Tpc.111.085183

Galea, C. A., Nourse, A., Wang, Y., Sivakolundu, S. G., Heller, W. T., and Kriwacki, R. W. (2008). Role of intrinsic flexibility in signal transduction mediated by the cell cycle regulator, p27 Kip1. J. Mol. Biol. 376, 827-838. doi: 10.1016/J.Jmb.2007.12.016

Garay-Arroyo, A., Colmenero-Flores, J. M., Garciarrubio, A., and Covarrubias, A. A. (2000). Highly hydrophilic proteins in prokaryotes and eukaryotes are common during conditions of water deficit. J. Biol. Chem. 275, 5668-5674. doi: $10.1074 /$ jbc. 275.8 .5668
Hanin, M., Brini, F., Ebel, C., Toda, Y., Takeda, S., and Masmoudi, K. (2011). Plant dehydrins and stress tolerance: versatile proteins for complex mechanisms. Plant Signal. Behav. 6, 1503-1509. doi: 10.4161/psb.6.10.17088

Hara, M. (2010). The multifunctionality of dehydrins: an overview. Plant Signal. Behav. 5, 503-508. doi: 10.4161/psb. 11085

Hara, M., Fujinaga, M., and Kuboi, T. (2005). Metal binding by citrus dehydrin with histidine-rich domains. J. Exp. Bot. 56, 2695-2703. doi: 10.1093/Jxb/ Eri262

Hara, M., Shinoda, Y., Tanaka, Y., and Kuboi, T. (2009). DNA binding of citrus dehydrin promoted by zinc ion. Plant Cell Environ. 32, 532-541. doi: 10.1111/J.1365-3040.2009.01947.X

Hara, M., Terashima, S., and Kuboi, T. (2001). Characterization and cryoprotective activity of cold-responsive dehydrin from Citrus unshiu. J. Plant Physiol. 158, 1333-1339. doi: 10.1078/0176-1617-00600

Hincha, D. K., and Thalhammer, A. (2012). LEA proteins: IDPs with versatile functions in cellular dehydration tolerance. Biochem. Soc. Trans. 40, 1000-1003. doi: 10.1042/Bst20120109

Houde, M., Daniel, C., Lachapelle, M., Allard, F., Laliberte, S., and Sarhan, F. (1995). Immunolocalization of freezing-tolerance-associated proteins in the cytoplasm and nucleoplasm of wheat crown tissues. Plant J. 8, 583-593. doi: 10.1046/J.1365313x.1995.8040583.X

Hughes, S., and Graether, S. P. (2011). Cryoprotective mechanism of a small intrinsically disordered dehydrin protein. Protein Sci. 20, 42-50. doi: 10.1002/pro.534

Hughes, S. L., Schart, V., Malcolmson, J., Hogarth, K. A., Martynowicz, D. M., Tralman-Baker, E., et al. (2013). The importance of size and disorder in the cryoprotective effects of dehydrins. Plant Physiol. 163, 1376-1386. doi: 10.1104/pp.113.226803

Hundertmark, M., and Hincha, D. K. (2008). LEA (late embryogenesis abundant) proteins and their encoding genes in Arabidopsis thaliana. BMC Genomics 9:118. doi: 10.1186/1471-2164-9-118

Ismail, A. M., Hall, A. E., and Close, T. J. (1999). Purification and partial characterization of a dehydrin involved in chilling tolerance during seedling emergence of cowpea. Plant Physiol. 120, 237-244. doi: 10.1104/pp.120.1.237

Jeffery, C. J. (2003). Moonlighting proteins: old proteins learning new tricks. Trends Genet. 19, 415-417. doi: 10.1016/S0168-9525(03)00167-7

Kazuoka, T., and Oeda, K. (1994). Purification and characterization of Cor85oligomeric complex from cold-acclimated spinach. Plant Cell Physiol. 35, 601-611.

Kim, S. Y., and Nam, K. H. (2010). Physiological roles of ERD10 in abiotic stresses and seed germination of Arabidopsis. Plant Cell Rep. 29, 203-209. doi: 10.1007/S00299-009-0813-0

Koag, M. C., Fenton, R. D., Wilkens, S., and Close, T. J. (2003). The binding of maize DHN1 to lipid vesicles. Gain of structure and lipid specificity. Plant Physiol. 131, 309-316. doi: 10.1104/Pp.011171

Koag, M. C., Wilkens, S., Fenton, R. D., Resnik, J., Vo, E., and Close, T. J. (2009). The K-segment of maize DHN1 mediates binding to anionic phospholipid vesicles and concomitant structural changes. Plant Physiol. 150, 1503-1514. doi: 10.1104/pp.109.136697

Kruger, C., Berkowitz, O., Stephan, U. W., and Hell, R. (2002). A metal-binding member of the late embryogenesis abundant protein family transports iron in the phloem of Ricinus communis L. J. Biol. Chem. 277, 25062-25069. doi: 10.1074/Jbc.M201896200

Kushwaha, R., Downie, A. B., and Payne, C. M. (2013). Uses of phage display in agriculture: sequence analysis and comparative modeling of late embryogenesis abundant client proteins suggest protein-nucleic acid binding functionality. Comput. Math. Methods Med. 2013, 470390. doi: 10.1155/2013/470390

Kushwaha, R., Lloyd, T. D., Schafermeyer, K. R., Kumar, S., and Downie, A. B. (2012). Identification of late embryogenesis abundant (LEA) protein putative interactors using phage display. Int. J. Mol. Sci. 13, 6582-6603. doi: 10.3390/Ijms13066582

Lin, C. T., and Thomashow, M. F. (1992). A cold-regulated Arabidopsis gene encodes a polypeptide having potent cryoprotective activity. Biochem. Biophys. Res. Commun. 183, 1103-1108. doi: 10.1016/S0006-291x(05)80304-3

Manfre, A. J., Lanni, L. M., and Marcotte, W. R. (2006). The Arabidopsis group 1 LATE EMBRYOGENESIS ABUNDANT protein AtEM6 is required for normal seed development. Plant Physiol. 140, 140-149. doi: 10.1104/Pp.105. 072967

Momma, M., Kaneko, S., Haraguchi, K., and Matsukura, U. (2003). Peptide mapping and assessment of cryoprotective activity of $26 / 27-\mathrm{kDa}$ dehydrin from soybean seeds. Biosci. Biotechnol. Biochem. 67, 1832-1835. doi: 10.1271/bbb.67.1832 
Mouillon, J. M., Eriksson, S. K., and Harryson, P. (2008). Mimicking the plant cell interior under water stress by macromolecular crowding: disordered dehydrin proteins are highly resistant to structural collapse. Plant Physiol. 148, 1925-1937. doi: 10.1104/pp.108.124099

Nakayama, K., Okawa, K., Kakizaki, T., Honma, T., Itoh, H., and Inaba, T. (2007). Arabidopsis Cor15am is a chloroplast stromal protein that has cryoprotective activity and forms oligomers. Plant Physiol. 144, 513-523. doi: 10.1104/pp.106.094581

Olvera-Carrillo, Y., Campos, F., Reyes, J. L., Garciarrubio, A., and Covarrubias, A. A. (2010). Functional analysis of the group 4 late embryogenesis abundant proteins reveals their relevance in the adaptive response during water deficit in Arabidopsis. Plant Physiol. 154, 373-390. doi: 10.1104/pp.110.158964

Olvera-Carrillo, Y., Reyes, J. L., and Covarrubias, A. A. (2011). Late embryogenesis abundant proteins: versatile players in the plant adaptation to water limiting environments. Plant Signal. Behav. 6, 586-589. doi: 10.4161/psb.6.4.15042

Pancsa, R., and Tompa, P. (2012). Structural disorder in eukaryotes. PLoS ONE 7:e34687. doi: 10.1371/journal.pone.0034687

Pufall, M. A., Lee, G. M., Nelson, M. L., Kang, H. S., Velyvis, A., Kay, L. E., et al. (2005). Variable control of Ets-1 DNA binding by multiple phosphates in an unstructured region. Science 309, 142-145. doi: 10.1126/Science.1111915

Rahman, L. N., Smith, G. S., Bamm, V. V., Voyer-Grant, J. A., Moffatt, B. A., Dutcher, J. R., et al. (2011). Phosphorylation of Thellungiella salsuginea dehydrins TsDHN-1 and TsDHN-2 facilitates cation-induced conformational changes and actin assembly. Biochemistry 50, 9587-9604. doi: 10.1021/bi201205m

Reyes, J. L., Campos, F., Wei, H., Arora, R., Yang, Y. I., Karlson, D. T., et al. (2008). Functional dissection of hydrophilins during in vitro freeze protection. Plant Cell Environ. 31, 1781-1790. doi: 10.1111/J.1365-3040.2008.01879.X

Reyes, J. L., Rodrigo, M. J., Colmenero-Flores, J. M., Gil, J. V., Garay-Arroyo, A., Campos, F., et al. (2005). Hydrophilins from distant organisms can protect enzymatic activities from water limitation effects in vitro. Plant Cell Environ. 28, 709-718. doi: 10.1111/J.1365-3040.2005.01317.X

Romero, P., Obradovic, Z., and Dunker, K. (1997). Sequence data analysis for long disordered regions prediction in the calcineurin family. Genome Inform. Ser. Workshop Genome Inform. 8, 110-124.

Rorat, T. (2006). Plant dehydrins - tissue location, structure and function. Cell. Mol. Biol. Lett. 11, 536-556. doi: 10.2478/s11658-006-0044-0

Ruibal, C., Salamo, I. P., Carballo, V., Castro, A., Bentancor, M., Borsani, O., et al. (2012). Differential contribution of individual dehydrin genes from Physcomitrella patens to salt and osmotic stress tolerance. Plant Sci. 190, 89-102. doi: 10.1016/j.plantsci.2012.03.009

Saavedra, L., Svensson, J., Carballo, V., Izmendi, D., Welin, B., and Vidal, S. (2006). A dehydrin gene in Physcomitrella patens is required for salt and osmotic stress tolerance. Plant J. 45, 237-249. doi: 10.1111/J.1365-313x.2005.02603.X

Sanchez-Ballesta, M. T., Rodrigo, M. J., LaFuente, M. T., Granell, A., and Zacarias, L. (2004). Dehydrin from Citrus, which confers in vitro dehydration and freezing protection activity, is constitutive and highly expressed in the flavedo of fruit but responsive to cold and water stress in leaves. J. Agric. Food Chem. 52, 1950-1957. doi: 10.1021/Jf035216

Soulages, J. L., Kim, K., Arrese, E. L., Walters, C., and Cushman, J. C. (2003). Conformation of a group 2 late embryogenesis abundant protein from soybean. Evidence of poly (L-proline)-type II structure. Plant Physiol. 131, 963-975. doi: 10.1104/Pp.015891

Sun, X. L., Rikkerink, E. H. A., Jones, W. T., and Uversky, V. N. (2013). Multifarious roles of intrinsic disorder in proteins illustrate its broad impact on plant biology. Plant Cell 25, 38-55. doi: 10.1105/Tpc.112.106062

Tantos, A., Friedrich, P., and Tompa, P. (2009). Cold stability of intrinsically disordered proteins. FEBS Lett. 583, 465-469. doi: 10.1016/J.Febslet.2008.12.054

Tompa, P., and Fuxreiter, M. (2008). Fuzzy complexes: polymorphism and structural disorder in protein-protein interactions. Trends Biochem. Sci. 33, 2-8. doi 10.1016/J.Tibs.2007.10.003

Tompa, P., and Kovacs, D. (2010). Intrinsically disordered chaperones in plants and animals. Biochem. Cell Biol. 88, 167-174. doi: 10.1139/O09-163

Tunnacliffe, A., and Wise, M. J. (2007). The continuing conundrum of the LEA proteins. Naturwissenschaften 94, 791-812. doi: 10.1007/S00114-007-0254-Y

Wang, Y. F., Fisher, J. C., Mathew, R., Ou, L., Otieno, S., Sublet, J., et al. (2011). Intrinsic disorder mediates the diverse regulatory functions of the Cdk inhibitor p21. Nat. Chem. Biol. 7, 214-221. doi: 10.1038/Nchembio.536

Wisniewski, M., Webb, R., Balsamo, R., Close, T. J., Yu, X. M., and Griffith, M. (1999). Purification, immunolocalization, cryoprotective, and antifreeze activity of PCA60: a dehydrin from peach (Prunus persica). Physiol. Plant. 105, 600-608. doi: 10.1034/J.1399-3054.1999.105402.X

Xie, C., Zhang, R. X., Qu, Y. T., Miao, Z. Y., Zhang, Y. Q., Shen, X. Y., et al. (2012). Overexpression of MtCAS31 enhances drought tolerance in transgenic Arabidopsis by reducing stomatal density. New Phytol. 195, 124-135. doi: 10.1111/J.1469-8137.2012.04136.X

Conflict of Interest Statement: The authors declare that the research was conducted in the absence of any commercial or financial relationships that could be construed as a potential conflict of interest.

Received: 01 July 2014; accepted: 09 October 2014; published online: 29 October 2014. Citation: Cuevas-Velazquez CL, Rendón-Luna DF and Covarrubias AA (2014) Dissecting the cryoprotection mechanisms for dehydrins. Front. Plant Sci. 5:583. doi: 10.3389/fpls.2014.00583

This article was submitted to Plant Physiology, a section of the journal Frontiers in Plant Science.

Copyright (c) 2014 Cuevas-Velazquez, Rendón-Luna and Covarrubias. This is an openaccess article distributed under the terms of the Creative Commons Attribution License (CC BY). The use, distribution or reproduction in other forums is permitted, provided the original author(s) or licensor are credited and that the original publication in this journal is cited, in accordance with accepted academic practice. No use, distribution or reproduction is permitted which does not comply with these terms. 